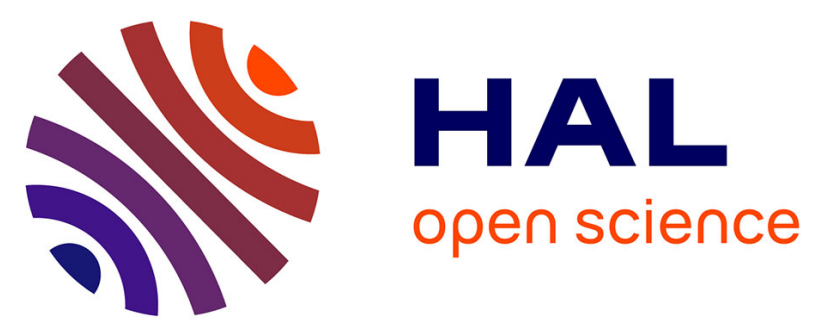

\title{
On the use of virtual ground scatterers to localize double and triple bounce scattering mechanisms for bistatic SAR
}

Ludovic Villard, P. Borderies

\section{- To cite this version:}

Ludovic Villard, P. Borderies. On the use of virtual ground scatterers to localize double and triple bounce scattering mechanisms for bistatic SAR. Journal of Electromagnetic Waves and Applications, 2015, 29 (5), pp.626-635. 10.1080/09205071.2015.1012594 . hal-01488145

\author{
HAL Id: hal-01488145 \\ https://hal.science/hal-01488145
}

Submitted on 12 Nov 2021

HAL is a multi-disciplinary open access archive for the deposit and dissemination of scientific research documents, whether they are published or not. The documents may come from teaching and research institutions in France or abroad, or from public or private research centers.
L'archive ouverte pluridisciplinaire HAL, est destinée au dépôt et à la diffusion de documents scientifiques de niveau recherche, publiés ou non, émanant des établissements d'enseignement et de recherche français ou étrangers, des laboratoires publics ou privés.

\section{(ㄷ)(1) $\$$}

Distributed under a Creative Commons Attribution - NonCommerciall 4.0 International 


\title{
On the use of virtual ground scatterers to localize double and triple bounce scattering mechanisms for bistatic SAR
}

\author{
L. Villard ${ }^{\mathrm{a} *}$ and P. Borderies ${ }^{\mathrm{b}}$ \\ ${ }^{a}$ CESBIO (Centre d'Études Spatiales de la Biosphère), 18 av. Edouard Belin, 31401 Toulouse, \\ France; ${ }^{b}$ ONERA (Office National d'Études et de Recherches Arospatiales), 2 av. Edouard Belin, \\ 31055 Toulouse, France
}

\begin{abstract}
In Radar remote sensing, the electromagnetic (em) modeling of scattering contributions from natural or man-made scatterers has often to deal with coupling scattering mechanisms due to the underlying reflecting surface (ground or water). For the monostatic configuration, this contribution is commonly associated with the so-called double bounce scattering mechanism, which is also known to be located in synthetic aperture radar (SAR) images at the vertical projection of the scatterer onto the ground. To explore this phenomenon in the more general case of bistatic SAR but also for triple bounce scattering, a new formalism using virtual scatterers is introduced in this paper. Based upon image theory and on far-field assumption, these virtual points are defined by simple interaction scatterers located on the reflecting surface, and involving the same traveling wave path than the considered scattering mechanism. Depending only on the incidence and scattering angles, elegant formulae in their simplicity are highlighted to construct these fictitious scatterers. The resulting reduction of multiple to simple interaction scattering mechanisms simplifies the finding of their location within bistatic SAR images, generalizing thereby migration effects associated to coupling mechanisms in the bistatic configuration.
\end{abstract}

Keywords: electromagnetic (em) modeling; double and triple bounce scattering mechanisms; image theory; bistatic synthetic aperture Radar (SAR)

\section{Introduction}

Electromagnetic (em) scattering from media made of discrete scatterers over an infinite reflecting surface has originated an abundant literature, especially in Radar remote sensing for the modeling of scattering from natural lands (see for instance [1]). Considering a discrete medium, the Distorted Born Wave Approximation (DBWA) is commonly applied as long as its volume fraction (volume of scatterers per unit volume within the medium) does not exceed values about $5 \%$ (cf. [2,3]). In agreement with this one-order approximation, incident or scattered field on or from a scatterer accounts for the direct path together with the averaged contribution of all the scatterers surrounding that path. The most common formulation of this averaged contribution results from the Forward Scattering Theorem as an application of the stationary phase theorem (cf. [4,5]), and leads to the famous 'Foldy' approximation, also referred to as the Effective field approximation. With regard to modeling of natural land surfaces such as agriculture fields or forests, this approximation is broadly used, especially for discrete and coherent (i.e. phase preserving) models such as those detailed in [6-9].

*Corresponding author. Email: ludovic.villard@cesbio.cnes.fr 
To account for the coupling effect with the underlying ground surface - which is modeled as an infinite horizontal plane - the specular point theory (SPT) is commonly used (cf. [10]). As described hereinafter, points on which specular reflections onto the ground hold are found using mirroring symmetry with respect to the reflecting surface, these specular points being intrinsic to double and triple bounce scattering mechanisms. At this point, it can be stressed that these restrictions (in the number of interactions accounted for) are not only determined by the growing complexity of these em scattering contributions but also by their isotropy essential for standard SAR compression methods (see e.g. [11]), whether in the common monostatic configuration or in the bistatic one, characterized by a distance between Transmitter and Receiver of the order of their distance to the observed scene (see e.g. [12]). For both kind of SAR configurations, the stability of the scattering return for each Radar pulse recorded along the acquisition long or slow-time axis - also called azimuth axis but possibly ambiguous in bistatic geometries - is required in order to get a standard point compression and retrieve the scattering contribution from the target. Hence, the limitations in our study of quasi-isotropic coupling terms (i.e. sufficiently stable to be properly retrieved by standard SAR compression) implying thereby quasi-isotropic scatterers and specular interactions onto the reflecting surface. Higher order and specific coupling terms such as those theoretically detailed in [13] or experimentally analyzed in [14] will be therefore left aside.

The question that arises in the following lies on the migration of scatterers involved in double and triple bounce scattering in bistatic SAR images. Such question is all the more challenging that severe image distortions may occur in bistatic geometries (cf. [15]) and that issues related to the so-called ghost scatterers associated with multiple scattering still give rise to many studies in the monostatic case (cf. [16]), particularly with respect to their stability as persistent scatterers (e.g. [17]). Given the complexity of multiple scattering, the possibility of finding virtual scatterers involving only one interaction together with the same traveling wave path (and thereby the same phase history) as the original higher order scattering mechanism would thus be very interesting. This is precisely the aim of the following developments, considering on the one hand the image theory framework and on the second hand plane wave fronts rather than spherical ones (hereinafter referred to as far-field approximation).

To introduce the method and its related hypothesis, definitions related to the bistatic configuration are given in the next section with the straightforward case a single reflection off a volume scatterer. Sections 3 and 4 are then dedicated to double and triple bounce interactions, while resulting applications are discussed in the concluding section.

\section{Problem definition considering simple scattering}

To introduce the problem and the notations used hereinafter, the straightforward case of a single bistatic reflection - that bounces off a volume scatterer - is considered in this section, as shown in Figure 1. As opposed to ground scatterers, the vertical position of a volume scatterer ' $\mathrm{P}$ ' is defined by a non-zero height noted ' $\mathrm{h}$ '. Ground or volume scatterers are considered as coherent targets, for which scattering contributions seem to come from individual points corresponding to their phase centers. The question at issue lies in finding the position of a virtual ground scatterer which involves the same traveling wave path than the simple bistatic reflection onto the considered volume scatterer.

In the following, a vector from point $\mathrm{P}$ to $\mathrm{T}$ is described by the symbol $\overrightarrow{P T}$, distance from $\mathrm{P}$ to $\mathrm{T}$ by $\overline{P T}$, and an unitary vector by $\hat{\mathbf{u}}$ so that $|\hat{\mathbf{P T}}|=|\overrightarrow{P T} / \overline{P T}|=1$. Concerning bistatic geometry, this study will be restricted to plane configurations, i.e. for every position 


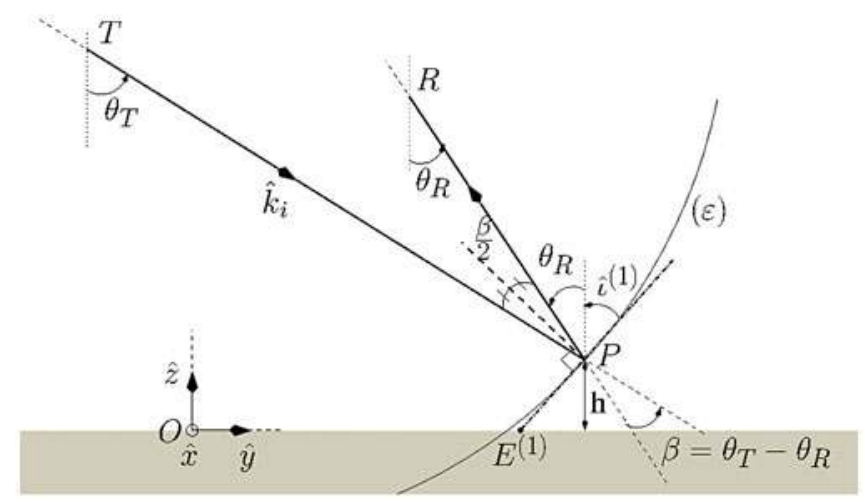

Figure 1. Virtual ground scatterer noted $E^{(1)}$ in the case of a single bistatic interaction, considering the em emitted wave for transmitter $\mathrm{T}$ which bounces off the volume scatterer $\mathrm{P}$ above the ground and then towards receiver R. Assuming that the ellipsoid iso-range $(\varepsilon)$ can be approximated by its tangent, the position of $E^{(1)}$ is given by the intersection between the ground and the tangent driven by the angle $\widehat{\iota}^{(1)}$.

of the receiver within the plane defined by $\hat{k}_{i}$ and $\hat{z}$, being, respectively, the unitary incident wave vector and unitary vertical one. The $3 \mathrm{D}$ generalization would indeed overwhelm the problem with geometrical features which may be not appropriated to keep the line of this letter. Following that restriction, a bistatic configuration is then completely defined through the plane coordinates of the transmitter and receiver noted ' $T$ ' and ' $R$ ', respectively. Considering the volume scatterer $\mathrm{P}$ (see Figure 1), the incidence and scattering angles $\theta_{\mathrm{T}}$ and $\theta_{\mathrm{R}}$ can be emphasized as well as the iso-range loci for the distance $|T P+P R|$, which turns out to be the ellipsoid $(\varepsilon)$ of foci T and R (cf. $[15,18]$ ), as the generalization to bistatic configuration of the spherical iso-ranges in the monostatic case. At that stage, it can be noted that the bistatic angle noted and defined by $\beta=\left(\theta_{\mathrm{T}}-\theta_{\mathrm{R}}\right)$ does not fully characterize the bistatic configuration, in particular since a zero bistatic angle may be associated to a non-monostatic geometry, when $\overline{P T} \neq \overline{P R}$. Besides, whether for airborne or spaceborne configurations, the height noted $h$ of a scatterer above the ground turns out to be sufficiently small in comparison to $\overline{P T}$ or $\overline{P R}$ so that the ellipsoid line passing through $\mathrm{P}$ can be approximated by its tangent, which intersects with the ground giving the projection into the corresponding pixel of all the points belonging to the same iso-range $|T P+P R|$. This intersection defines the equivalent ground point for a single interaction onto a volume scatter, noted $E^{(1)}$. To make its location explicit, one can recall the well-known property of the tangent to an ellipsoid which turns out to be normal to the bisectrix vector $\hat{\beta}$ at the tangent point, that is:

$$
\frac{d(\overrightarrow{O P})}{d t} \cdot(\hat{\mathbf{P T}}+\hat{\mathbf{P R}})=\frac{d(\overrightarrow{O P})}{d t} \cdot \vec{\beta}=0
$$

with $t$ as a real number used to parametrize the vector $\overrightarrow{O P}(t)$. Having in mind this property, it follows that $E^{(1)}$ can be found from the angle $\widehat{\iota}^{(1)}$ between the tangent to the ellipsoid and the vertical axis, with:

$$
\widehat{\iota}^{(1)}=\frac{\pi}{2}-\left(\beta / 2+\theta_{\mathrm{R}}\right)=\frac{\pi}{2}-\frac{\theta_{\mathrm{T}}+\theta_{\mathrm{R}}}{2}
$$


As a result, this characterization can be considered as a generalization in the bistatic configuration of the well-known layover effect in monostatic SAR images, truly intrinsic to the slant range geometry of Radar acquisitions, unlike optical ones at the nadir of the observed scene which do not suffer from that effect. Indeed, in reducing the 3D scene information through the projection on the ground of all the scatterers belonging to the same iso-range or pixel, the layover effect originates image distortions (e.g. the mixed-up between scattering contributions from several targets) and is thereby essential to account for in monostatic or bistatic SAR imaging.

\section{Virtual ground scatterer for double bounce scattering mechanism}

\subsection{Monostatic case}

Even though the monostatic case constitutes a particular bistatic configuration which could have been combined with the next subsection, the developments given hereinafter have not been skipped since it emphasizes the most common case in Radar remote sensing and makes the following geometrical calculations more comprehensive.

Within the specular point theory framework (detailed in introduction), the points onto the ground where the specular reflections hold can be judiciously found using the mirroring principle. As shown in Figure 2, considering a given scatterer $P$ and its mirror symmetric noted $P_{\aleph}$, the specular points are determined by the intersections between the ground surface and the lines $\left(T P_{\aleph}\right)$ and $\left(R P_{\aleph}\right)$ - joining $P_{\aleph}$ with $\mathrm{T}$ and $\mathrm{R}$, respectively. Since $\mathrm{T}$ and $\mathrm{R}$ coincide in the monostatic configuration, the specular points onto the ground noted $\left(G^{\mathrm{T}}\right.$ and $G^{\mathrm{R}}$ ) are located at the same position so that the monostatic double bounce is made of two identical paths (with yet opposite time directions), originating thereby coherent effects (superposition of two waves in phase) commonly referred to as the backscattering enhancement (see for instance [19]). In this subsection, $\mathrm{T}$ and $G^{\mathrm{T}}$ have been arbitrary preferred.

The following two-step demonstration to determine $E^{(2)}-$ the point on which a single interaction involves the same traveling wave path as the double bounce scattering mechanism associated to $\mathrm{P}$ - will first consist in showing the existence of $E^{(2)}$ and then its uniqueness. Let us then consider the configuration presented in Figure 2 with $E^{(2)}$ as the vertical projection of $\mathrm{P}$ onto the ground. According to the afore-mentioned hypothesis of plane wave fronts ( $\mathrm{P}$ being sufficiently far for $\mathrm{T}$ and $\mathrm{R}$ ), it can be stated that:

$$
\begin{aligned}
2 \overline{E_{1} E^{(2)}} & =\overline{E_{1} E_{2}}+2 \overline{E_{2} E^{(2)}}+\overline{E_{2}^{\prime} G^{\mathrm{T}}} \\
& =\overline{E_{1}^{\prime} P}+\overline{P_{\aleph} E_{2}^{\prime}}+\overline{E_{2}^{\prime} G^{\mathrm{T}}} \\
& =\overline{E_{1}^{\prime} P}+\overline{P_{\aleph} G^{\mathrm{T}}} \\
& =\overline{E_{1}^{\prime} P}+\overline{P G^{\mathrm{T}}}
\end{aligned}
$$

where equality $2 \overline{E_{2} E^{(2)}}=\overline{P_{\aleph} E_{2}^{\prime}}$ results from the Thales' theorem (or also called intercept theorem) applied to the triangle $\left(G^{\mathrm{T}} P_{\aleph} P\right)$ with $\left(G^{\mathrm{T}} P_{\aleph}\right) / /\left(E_{1} E^{(2)}\right)$ and $\overline{P P_{\aleph}}=2 \overline{P E^{(2)}}$, and where equality $\overline{G^{\mathrm{T}} P}=\overline{G^{\mathrm{T}} P_{\aleph}}$ results from construction of $P_{\aleph}$. From Equation (2), it follows that

$$
2 \cdot \overline{T E^{(2)}}=\overline{T P}+\overline{P G^{\mathrm{T}}}+\overline{G^{\mathrm{T}} T}
$$

which proves the existence condition of $E^{(2)}$. Let's now suppose that equality in (3) is verified by two points $E^{(2)} A$ and $E^{(2)} B$. By definition, the simple interaction onto these two points involves the same traveling wave path, so that $E^{(2)}{ }_{A}$ and $E^{(2)}{ }_{B}$ belong to the 


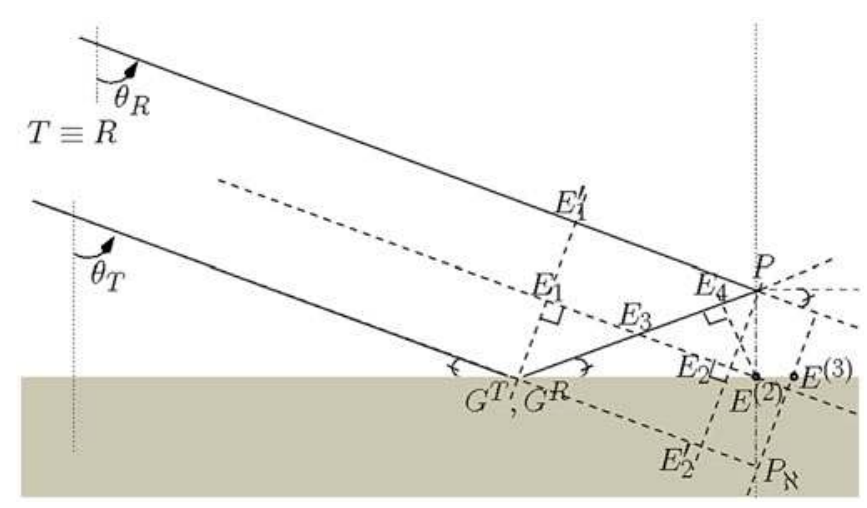

Figure 2. Monostatic configuration for which transmitter $\mathrm{T}$ and receiver $\mathrm{R}$ coincide, as well as the specular ground points $G^{\mathrm{T}}$ and $G^{\mathrm{R}}$. The virtual ground points for the double and triple bounce scattering mechanisms are, respectively, noted $E^{(2)}$ and $E^{(3)}$.

same spherical iso-range which intersects with the ground giving a single point, hence the uniqueness condition for point $E^{(2)}$.

Alternatively, it results from the Thales' theorem applied to in the triangles $\left(G^{\mathrm{T}} P E_{1}^{\prime}\right)$ and $\left(G^{\mathrm{T}} P_{\aleph} P\right)$ that:

$$
\begin{aligned}
& 2 \cdot \overline{E_{1} E_{3}}=\overline{E_{1}^{\prime} P} \quad \text { and } \quad 2 \cdot \overline{E_{3} E^{(2)}}=\overline{G^{\mathrm{T}} P_{\aleph}}=\overline{G^{\mathrm{T}} P} \\
& \text { hence: } \quad 2 \cdot \overline{E_{1} E^{(2)}}=2 \cdot \overline{E_{1} E_{3}}+2 \cdot \overline{E_{3} E^{(2)}} \\
& =\overline{E_{1}^{\prime} P}+\overline{G^{\mathrm{T}} P}
\end{aligned}
$$

which also proves the existence condition for $E^{(2)}$.

Finally, a last demonstration can be also put forward since it will be used in the subsequent paragraph in the bistatic case: from the construction of points $E_{1}$ and $E_{4}$, it results that:

$$
\overline{E_{1} E_{2}}=\overline{G^{\mathrm{T}} E_{4}} \text { and } \overline{E_{2} E^{(2)}}=\overline{E_{4} P}
$$

hence the term by term equality:

$$
\overline{G^{\mathrm{T}} E_{4}}+\overline{E_{4} P}+\overline{P E_{1}^{\prime}}=\overline{E_{1} E^{(2)}}+\overline{E^{(2)} E_{2}}+\overline{E_{2} E_{1}}
$$

which constitutes another necessary condition for the localization of $E^{(2)}$.

Using these developments as an introduction to more advanced cases, the well-known vertical projection on the ground of the double bounce backscattering mechanism has been rewritten. This phenomenon is indeed very common in monostatic SAR imaging: whereas the position of a given scatterer will be shifted according to the layover effect, its double bounce contribution will remain at its vertical projection, whatever the considered scatterer height above the ground.

At that stage, we can already infer that $\widehat{\iota}^{(2)}-$ the angle between $\widehat{P E^{(2)}}$ and $\hat{z}-$ is likely to be proportional to the bistatic angle $\beta$, so that $\beta$ nullifies $\widehat{\iota}^{(2)}$ in the monostatic case. 


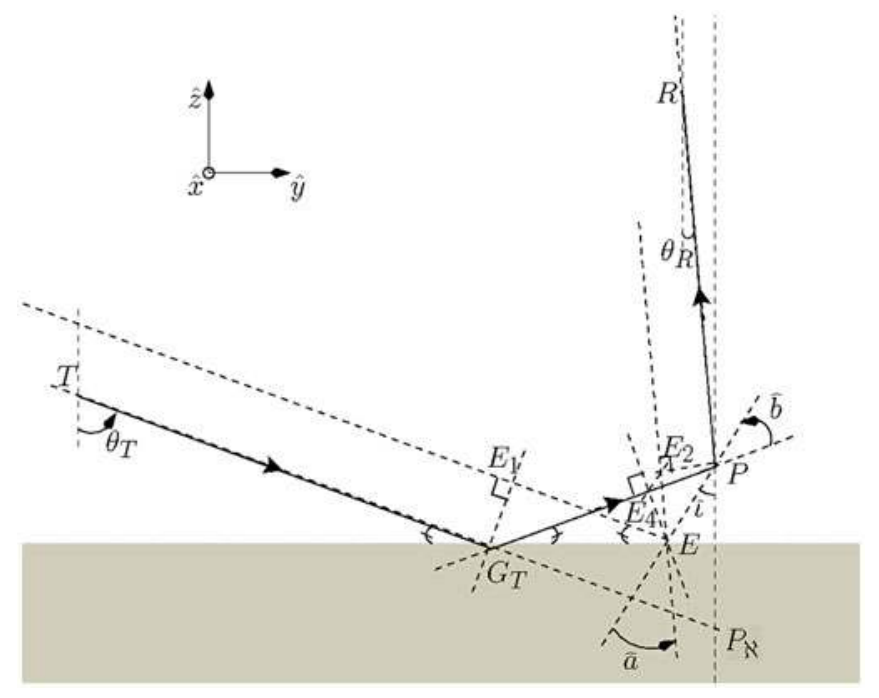

Figure 3. Traveling wave path in the bistatic configuration for the double bounce scattering mechanism with respect to the transmitter T. The virtual ground point $E^{(2)}$ corresponds to the position of the floating point $E$ onto the ground so that: $\mathcal{L}(T \rightarrow E \rightarrow R) \underset{E=E^{(2)}}{=} \mathcal{L}\left(T \rightarrow G^{\mathrm{T}} \rightarrow P \rightarrow R\right)$.

For the sake of clarity, $E^{(2)}$ is not displayed above but can be easily located, moving $E$ on the right so that $\hat{a}=\hat{b}$.

\subsection{Bistatic case within the plane of incidence}

As stated in Section 2, the bistatic configurations considered hereinafter are restricted to the plane of incidence, defined by the incidence wave vector $\hat{k}_{i}$ and the vertical vector $\hat{z}$. The geometry for such configuration is displayed in Figure 3, where an arbitrary point scatterer $P$ above the ground is chosen, as well as transmitter $\mathrm{T}$ and receiver $\mathrm{R}$, from now on located at different places. Still considering the specular point theory framework, specular ground points involved for the double bounce scattering mechanism are determined using the mirror symmetric point $P_{\aleph}$. Two different specular ground points noted $G^{\mathrm{T}}$ and $G^{\mathrm{R}}$ with respect to $\mathrm{T}$ and $\mathrm{R}$ can then be found, noticing that both do not coincide anymore as for monostatic cases.

As a result, two distinct equivalent ground points will be searched for, starting from the one with respect to the transmitter which is still noted $E^{(2)}$ in the following (as long as both are not involved). Geometrical features are given in Figure 3, especially for the construction of $G^{\mathrm{T}}$ based on $P_{\aleph}$. Dot lines are dedicated to virtual geometrical points (especially towards $E^{(2)}$ ) whereas plain lines are dedicated to the 'real' traveling wave path: $T \rightarrow G^{\mathrm{T}} \rightarrow P \rightarrow R$, whose length will be hereinafter noted with the operator $\mathcal{L}(\cdot)$.

Let's consider the angle $\widehat{\imath}$ between the vertical axis (along $\hat{\mathbf{z}}$ and the line passing by $P$ and the candidate floating point $E$, which position on the ground is sought to be $E^{(2)}$. Points $E_{1}$ and $E_{4}$ are constructed from the projection of $G^{\mathrm{T}}$ and $E$, respectively, so that whatever the position of $\mathrm{E}$ onto the ground, the triangle of base $G^{\mathrm{T}} E$ is isosceles and $\overline{E_{1} E}=\overline{G^{\mathrm{T}} E_{4}}$. Let us now emphasize the angles $\hat{a}$ and $\hat{b}$, respectively, defined in triangles $\left(E_{2} E P\right)$ and $\left(E P E_{4}\right)$ (still in Figure 3). These angles can thus be expressed as follows:

$$
\hat{a}=\theta_{\mathrm{R}}+\hat{\imath} \text { and } \hat{b}=\theta_{\mathrm{T}}-\widehat{\imath}
$$


With that construction, the equality $E E_{2}=E_{4} P$ holds if and only if the triangle of base $E P$ is isosceles, i.e. $\hat{a}=\hat{b}$. From that equality, it comes that:

$$
\overline{G^{\mathrm{T}} P}=\overline{G^{\mathrm{T}} E_{4}}+\overline{E_{4} P}=\overline{E_{1} E}+\overline{E E_{2}}
$$

As a result, with the starting assumption that both transmitter and receiver are far enough from $P$ to consider the equi-phase plane surfaces, a sufficient and necessary condition on the location of $E$ to verify $\mathcal{L}(T \rightarrow E \rightarrow R)=\mathcal{L}\left(T \rightarrow G^{\mathrm{T}} \rightarrow P \rightarrow R\right)$ lies in $\hat{a}=\hat{b}$, hence the following elegant formula in its simplicity for the virtual ground point $E^{(2)}$ associated to the double bounce scattering mechanism with respect to the transmitter, which turns out from (5) to be half the bistatic angle:

$$
\left.\widehat{\iota}^{(2)}\right|_{G^{T}}=\frac{\beta}{2}
$$

Concerning now the double bounce with respect to the receiver, the reciprocity of the configuration can be invoked: if $T$ and $R$ are inverted, $G^{\mathrm{R}}$ is located at the former position of $G^{\mathrm{T}}$ so we come back to the traveling wave path associated to the former double bounce with respect to $\mathrm{T}$ and then to the former virtual ground point $E^{(2)}$ and its associated angle $\widehat{\iota}^{(2)}$. As expected, reversing the situation by reciprocity does correspond to a switch between $G^{\mathrm{T}}$ and $G^{\mathrm{R}}$ as well as between $\mathrm{T}$ and $\mathrm{R}$ in formula (6), hence the following generalization:

$$
\widehat{\iota}^{(2)}= \pm \frac{\beta}{2}
$$

where the plus or minus are, respectively, for the double bounce with respect to the transmitter or to the receiver. From that result, it is interesting to note that depending on the largest angles between $\theta_{\mathrm{T}}$ and $\theta_{\mathrm{R}}$, the double bounce scattering mechanisms are either projected forward or backward in comparison with the monostatic case where both double bounces are vertically projected on the ground. Besides, this result also means that double bounce contributions (with respect to T or R) of all scatterers located along the line $\left(E^{(2)} P\right)$ will be associated with the same traveling distance or time, giving thereby the shapes of Radar isoranges for the scattering mechanisms concerned. Therefore, not only the geometrical phase for bistatic double bounces are different (unlike the monostatic superposition in phase) but these contributions may be not recorded in the same pixel, especially for SAR images at high (spatial) resolution.

\section{Virtual ground scatterer for triple bounce scattering mechanism}

The following developments are now dedicated to triple bounce scattering. To come up with the traveling path associated to that scattering mechanism, the coupling interactions between vegetation and ground scatterers are considered for both downwards and upwards paths, respectively from transmitter T towards an arbitrary volume scatterer (still noted $P$ ) and from $P$ to receiver R (see Figure 4). Still in agreement with the afore-invoked specular point theory, both specular ground reflections onto points $G^{\mathrm{T}}$ and $G^{\mathrm{R}}$ are thereby involved, being still constructed using $P_{\aleph}$, the mirror symmetric of $P$ (as in Section 3). Supported by geometrical details provided in Figure 4, the problem now at issue being in the location of the floating point $E$ onto the ground so that:

$$
\mathcal{L}(T \rightarrow E \rightarrow R) \underset{E=E^{(3)}}{=} \mathcal{L}\left(T \rightarrow G^{\mathrm{T}} \rightarrow P \rightarrow G^{\mathrm{R}} \rightarrow R\right)
$$




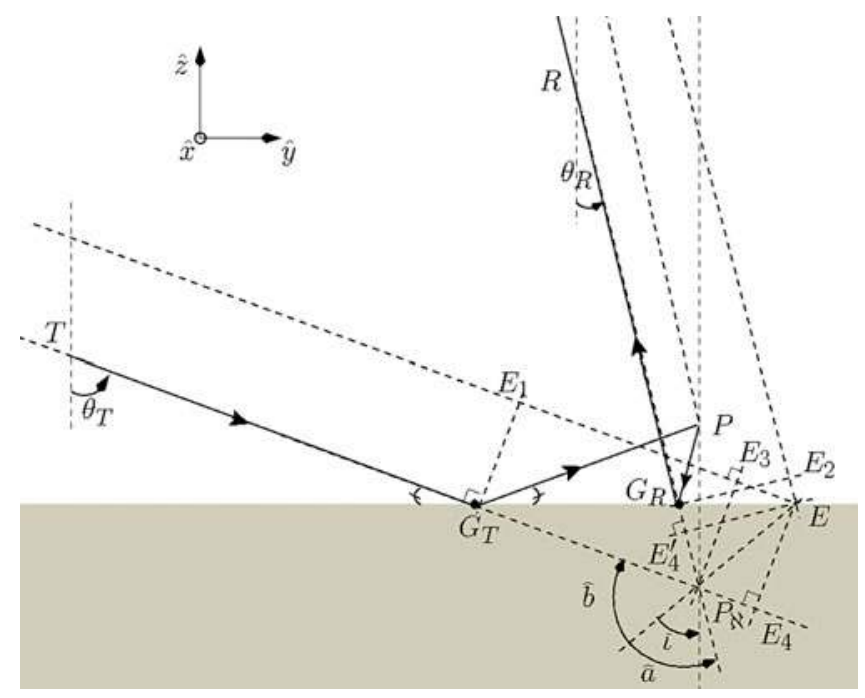

Figure 4. Traveling wave path in the bistatic configuration for the triple bounce scattering mechanism. The virtual ground point $E^{(3)}$ corresponds to the position of the floating point $E$ onto the ground so that: $\mathcal{L}(T \rightarrow E \rightarrow R) \underset{E=E^{(3)}}{=} \mathcal{L}\left(T \rightarrow G^{\mathrm{T}} \rightarrow P \rightarrow G^{\mathrm{R}} \rightarrow R\right)$.

In agreement with the previous hypothesis, equi-phase plane surfaces are still considered so that:

$$
\begin{aligned}
\mathcal{L}(T \rightarrow E \rightarrow R)- & \mathcal{L}\left(T \rightarrow G^{\mathrm{T}} \rightarrow P \rightarrow G^{\mathrm{R}} \rightarrow R\right) \\
& =\overline{E_{1} E}+\overline{E E_{2}}-\left(\overline{G^{\mathrm{T}} P}+\overline{P G^{\mathrm{R}}}\right) \\
& =\overline{E_{1} E_{3}}+\overline{E_{3} E}+\overline{E_{4}^{\prime} G^{\mathrm{R}}}-\left(\overline{G^{\mathrm{T}} P}+\overline{P G^{\mathrm{R}}}\right) \\
& =\overline{E_{1} E_{3}}+\overline{E_{3} E}+\overline{E_{4}^{\prime} G^{\mathrm{R}}}-\left(\overline{G^{\mathrm{T}} P_{\aleph}}+\overline{P_{\aleph} G^{\mathrm{R}}}\right) \\
& =\overline{E_{3} E}+\overline{E_{4}^{\prime} G^{\mathrm{R}}}-\overline{P_{\aleph} G^{\mathrm{R}}} \\
& =\overline{E_{3} E}-\overline{P_{\aleph} E_{4}^{\prime}}
\end{aligned}
$$

From the above equation, it can be deduced from the last line that equality (8) holds if $\overline{E_{3} E}=\overline{P_{\aleph} E_{4}^{\prime}}$, i.e. if the line $\left(P_{\aleph} E\right)$ bisects the corner $\left(E_{4}^{\prime} P_{\aleph} E_{4}\right)$, meaning that:

$$
\begin{aligned}
\widehat{a} & =\widehat{b} \\
\text { so that: } \theta_{\mathrm{R}}+\widehat{\imath} & =\pi-\theta_{\mathrm{T}}-\widehat{\imath}
\end{aligned}
$$

providing the sought formula:

$$
\widehat{\iota}^{(3)}=\frac{\theta_{\mathrm{T}}+\theta_{\mathrm{R}}}{2}-\frac{\pi}{2}=-\widehat{\iota}^{(1)}
$$

Existence condition for $E^{(3)}$ is thus proven by the above expression. In the event that two points $E_{A}^{(3)}$ and $E_{B}^{(3)}$ exist, these points onto which simple interactions occur would belong to the same ellipsoid iso-range, which intersects with the ground giving a single point, proving thereby the uniqueness condition for point $E^{(3)}$. 
As far as SAR applications are concerned, the magnitude of triple bounce scattering mechanism is more likely to be significant over built-up structures such as ships, bridges, or buildings (see for instance [16,20]) as well as flooded regions, where specular ground reflections are favored by flat and smooth surfaces. According to the previous result Equation (11), it is rather remarkable that whatever the scatterer above the ground, its triple bounce contribution will appear backwards, with exactly the opposite distance which shifts forward the volume contribution, generalizing thereby the layover effect to triple bounce scattering mechanism in bistatic configuration.

\section{Discussion on resulting applications \& conclusion}

In this paper, an elegant theoretical method to reduce double and triple bounce scattering mechanisms to a simple interaction onto a virtual ground scatterer is developed, for any bistatic configuration where the receiver belongs to the plane of incidence. This method is based on virtual scatterers located on the ground surface and for which the simple interaction involves the same traveling wave path as its associated multiple scattering mechanism. With respect to the original scatterer, the location on the ground of this virtual point turns out to be driven through remarkable formulae in their simplicity, involving simply half the signed bistatic angle in the case of double bounce scattering mechanism with opposite sign between double bounce with respect to transmitter or to receiver - and the opposite angle associated with the simple layover in the case of triple bounce scattering mechanism.

Beyond novelty of the theoretical formulation in SAR configurations for which we have little experience, these results offer a simple but efficient way to characterize the migration phenomenon in bistatic images and the so-called ghost scatterers, mostly due to multiple scattering. However, as for current state-of-the art monostatic SAR imaging methods, non-supervised corrections to avoid these ghost scatterers are not applicable without a-priori information about the observed scene. In addition, these results also demonstrate that the voxel shapes (i.e. the 3D volumes which encompass all the scatterers whose specific contribution falls into a given pixel) is intrinsic to each scattering mechanism, which is essential for analytical formulation in em modeling and especially to derive the interferometric phase sensitivity to scatterer height.

Being less and less limited by technological barriers (especially related to synchronisation), one can currently attest of a new resurgence of bistatic Radar, not only characterized by airborne concepts but also by hybrid configurations (like GNSS-Reflectometry) and the willing of putting bistatic configurations into space (e.g. with the SAOCOM-CS ESA candidate mission [21]). Fostered by these promising concepts, next steps will be dedicated to generalize our results to the full bistatic hemispherical space (combining geometrical features of both scattering and incident planes) and to specific SAR applications, in the case of non-stationary configurations and polarimetric and interferometric bistatic SAR acquisitions.

\section{Acknowledgements}

The authors are truly grateful to Thuy Le Toan, especially for its interest and support which ease the finalization of developments and writing. This study has been also made possible thanks to the financial support of CNES (Centre National d'Etudes Spatiales) through the TOSCA research program. 


\section{References}

[1] Ulaby F, Moore R, Fung A. Microwave remote sensing: active and passive. Vol. III, Volume scattering and emission theory of advanced systems and applications. Dedham (MA): Artech House; 1986.

[2] Tsang L, Kong JA, Shin R. Theory of microwave remote sensing. NewYork (NY): Wiley Interscience; 1985.

[3] Tsang L, Kong JA. Scattering of electromagnetic waves. Vol. 3, Advanced Topics. NewYork (NY): Wiley Interscience; 2001. p. 413.

[4] Ishimaru A. Wave propagation and scattering in random media. Vol. 2. New York (NY): Academic Press; 1978.

[5] Liao J. Foldy-lax approximation on multiple scattering by many small scatterers. Applicable Anal. 2013;92:2547-2560.

[6] Lin YC, Sarabandi K. A Monte Carlo coherent scattering model for forest canopies using fractalgenerated trees. IEEE Trans. Geosci. Remote Sens. 1999;37:440-451.

[7] Marliani F, Paloscia S, Pampaloni P, Kong J. Simulating coherent backscattering from crops during the growing cycle. IEEE Trans. Geosci. Remote Sens. 2002;40:162-177.

[8] Thirion L, Colin E, Dahon C. Capabilities of a forest coherent scattering model applied to radiometry, interferometry, and polarimetry at P- and L-band. IEEE Trans. Geosci. Remote Sens. 2006;44:849-862.

[9] Villard L. 2009. Forward and inverse modeling of bistatic SAR Observables: Applications in forest remote sensing [PhD Thesis] defended December 21, 2009. Toulouse, France: National School of Aeronautics and Space (ISAE-Supaero); achieved jointly at ONERA (the French Aerospace Lab, Toulouse) and DLR (German Aerospace Center, Oberpfaffenhofen).

[10] Beckmann P, Spizzichino A. The scattering of electromagnetic waves from rough surfaces. New York (NY): Pergamon; 1963.

[11] Bamler R. Principles of synthetic aperture radar. Surv. Geophys. 2000;21:147-157.

[12] Cherniakov M, Moccia A, Moreira A, Krieger G, D'Errico M. Bistatic radars: emerging technology. Birmingham: The University of Birmingham, John Wiley \& Sons; 2008.

[13] Van Zyl J. The Effect of topography on radar scattering from vegetated areas. IEEE Trans. Geosci. Remote Sens. 1993;31:153-160.

[14] Auer S, Gernhardt S, Bamler R. Ghost persistent scatterers related to multiple signal reflections. Geosci. Remote Sens. Lett. IEEE. 2011;8:919-923.

[15] Villard L, Hajnsek I, Borderies P, Papathanassiou K. Pol-InSAR simulations in forest bistatic scattering. In: Proceedings of the EUSAR 2008; 7th european conference on synthetic aperture radar. Seiten: VDE Publishing House; 2008. pp. 1-4.

[16] Auer S, Gernhardt S. Linear signatures in urban sar images - partly misinterpreted? Geosci. Remote Sens. Lett. IEEE. 2014;11:1762-1766.

[17] Margarit G, Mallorqui J, Pipia L. Polarimetric characterization and temporal stability analysis of urban target scattering. Geosci. Remote Sens. IEEE Trans. 2010;48:2038-2048.

[18] Villard L, Hajnsek I, Borderies P. 2008. Bistatic Pol-InSAR scenario \& evaluation by forest scattering simulations. In: International Geoscience and Remote Sensing Symposium (IGARSS); Vol. 4, Boston MA, USA: IEEE; p. 97-100.

[19] Saatchi S, McDonald K. Coherent effects in microwave backscattering models for forest canopies. IEEE Trans. Geosci. Remote Sens. 1997;35:1032-1044.

[20] Soergel U, Cadario E, Thiele A, Thoennessen U. Feature extraction and visualization of bridges over water from high-resolution insar data and one orthophoto. IEEE J. Sel. Top. Appl. Earth Obs. Remote Sens. 2008;1:147-153.

[21] Gebert N, Carnicero Dominguez B, Davidson MW, Diaz Martin MW, Silvestrin P. 2014. SAOCOM-CS - a passive companion to saocom for single-pass L-band sar interferometry. In: Proceedings of the EUSAR 2014; 10th European conference on synthetic aperture Radar; Seiten: VDE Publishing House. p. 1-4. 